\title{
On the Convergence of Input-Output Fuzzy Cognitive Maps
}

\author{
István Á. Harmati ${ }^{1(\varpi)}$ and László T. Kóczy ${ }^{2,3}$ \\ 1 Department of Mathematics and Computational Sciences, Széchenyi István \\ University, Egyetem tér 1, Győr 9026, Hungary \\ harmati@sze.hu \\ 2 Department of Information Technology, Széchenyi István University, \\ Egyetem tér 1, Győr 9026, Hungary \\ koczy@sze.hu \\ 3 Department of Telecommunication and Media Informatics, Budapest University \\ of Technology and Economics, Magyar tudósok körútja 2, Budapest 1117, Hungary
}

\begin{abstract}
Fuzzy cognitive maps are recurrent neural networks, where the neurons have a well-defined meaning. In certain models, some neurons receive outer input, while other neurons produce the output of the system. According to this observation, some neurons are categorized as input neurons and the others are the state neurons and output neurons. The output of the system is provided as a limit of an iteration process, which may converge to an equilibrium point, but limit cycles or chaotic behaviour may also show up. In this paper, we examine the existence and uniqueness of fixed points for two types of input-output fuzzy cognitive maps. Moreover, we use network-based measures like in-degree, out-degree and connectivity, to express conditions for the convergence of the iteration process.
\end{abstract}

Keywords: Fuzzy cognitive map • Input-output fuzzy cognitive map • Stability $\cdot$ Convergence $\cdot$ Equilibrium point

\section{Introduction}

Fuzzy cognitive maps (FCMs) are decision support tools, based on the recurrent neural network modelling method. The essence is that the neurons have welldefined meaning, they represent specific factors or characteristics of the modelled system [14]. The structure of a fuzzy cognitive map is a weighted, directed graph. The weights are assigned to the edges from the interval $[-1,1]$ to express the strength and direction of causal connections. The current states of the neurons (which are called concepts in FCM literature) are also described by values from the $[0,1]$ interval (or from the interval $[-1,1]$, see for example [15]). These are the activation values of the concepts [12].

The system can be described by the set of concepts $\left(C_{1}, C_{2}, \ldots, C_{n}\right)$; the current activation values of the concepts $\left(A_{1}, A_{2}, \ldots, A_{n}\right)$; the weight matrix 
$W$ which assigns weight $w_{i j}$ to each edge connecting the nodes $C_{i}$ and $C_{j}$, expressing how strongly influenced is concept $C_{i}$ by concept $C_{j}$. The sign of $w_{i j}$ indicates whether the relationship between $C_{j}$ and $C_{i}$ is direct or inverse. So matrix $W$ represents the weighted causal connections between the concepts. A transformation (or transfer, or threshold) function $f: \mathbb{R} \rightarrow[0,1]$ calculates the activation value of concepts at every time step of the iteration and keeps the activation values in the allowed range (sometimes a function $f: \mathbb{R} \rightarrow[-1,1]$ is applied).

The iteration rule which calculates the values of the concept at every step may or may not include self-feedback. In general form it can be written as

$$
A_{i}(k)=f\left(\sum_{j=1, j \neq i}^{n} w_{i j} A_{j}(k-1)+d_{i} A_{i}(k-1)\right)
$$

where $A_{i}(k)$ is the value of concept $C_{i}$ at discrete time $k, w_{i j}$ is the weight of the connection from concept $C_{j}$ to concept $C_{i}$ and $0 \leq d_{i} \leq 1$ expresses the possible self-feedback. If $d_{i}=0$, then there is no self-feedback. If we include the $d_{i}$ s into the diagonal of weight matrix $W$, the iteration equation can be rewritten in more compact style:

$$
A_{i}(k+1)=f\left(\sum_{j=1}^{n} w_{i j} A_{j}(k)\right)=f\left(w_{i} A(k)\right),
$$

where $w_{i}=\left[w_{i 1}, \ldots, w_{i n}\right]$ is the $i$ th row of $W$ and $A(k)=\left[A_{1}(k), \ldots, A_{n}(k)\right]^{T}$ is the concept vector after $k$ iterations. We apply dot product between them, so $w_{i} A^{(k)}$ is a real number.

Moreover, if we couple the coordinates of the concept vector together and denote by $G$ the mapping $\mathbb{R}^{n} \rightarrow \mathbb{R}^{n}$ that generates the concept vector $A(k+1)$ from $A(k)$, then we have that:

$$
A(k+1)=\left[\begin{array}{c}
A_{1}(k+1) \\
\vdots \\
A_{n}(k+1)
\end{array}\right]=\left[\begin{array}{c}
f\left(w_{1} A(k)\right) \\
\vdots \\
f\left(w_{n} A(k)\right)
\end{array}\right]=G(A(k)) .
$$

The iteration rule is repeated until either the FCM converges to an equilibrium state (fixed point) or the maximal number of iterations is reached. Mathematically, the FCM may converge to a fixed point, may arrive to a limit cycle or shows chaotic pattern $[4,10,11]$.

Sufficient mathematical condition for the existence and uniqueness of fixed points of a special class of FCMs has been introduced in [2], expressed by the sum of the squared elements of $W$. This result was later generalized in [4]. In [7], the authors examined the problem of unique fixed points taking into consideration only the topology of the FCM, but not the weights. They pointed out that if the parameter of the sigmoid transfer function is small enough, then the FCM has 
exactly one fixed point. In [8] the global asymptotic stability of FCMs has been discussed via Lyapunov method.

Recently, various generalizations of FCMs have been introduced $[1,3,9]$, where some concepts (neurons, nodes of the graph) are considered as inputs to the system, while some other (or all of the remaining) concepts form the output of the system. This article aims to provide sufficient converge conditions for these models, based on the weight structure and the parameter(s) of the transfer (threshold) function(s).

The rest of the paper is organized as follows. In Sect. 2 we recall the most important mathematical tools and notions applied in the proofs of our findings. In Sect. 3, we examine the behaviour of the generalized FCM model introduced in [1] and [3]: sufficient conditions for the existence and uniqueness of fixed points are provided. Moreover, we show that under certain mathematical conditions different input values may produce different steady-state concept vectors and different output values. In Sect.4, sufficient condition for the convergence of FCM model introduced in [9] is given, and finally in Sect. 5 we summarize the main contributions of the paper.

\section{Mathematical Background}

In this section, we recall the most important definitions and results applied in the subsequent sections. First we recall the definition of contraction mapping [13]:

Definition 1. Let $(X, d)$ be a metric space. A mapping $G: X \rightarrow X$ is a contraction mapping or contraction if there exists a constant $c$ (independent from $x$ and $y)$, with $0 \leq c<1$, such that

$$
d(G(x), G(y)) \leq c d(x, y) .
$$

The notion of contraction is related to the distance metric $d$ applied. It may happen that a function is a contraction w.r.t. one distance metric, but not a contraction w.r.t. another distance metric. The iterative process of an FCM may end at an equilibrium point, which is a so-called fixed point.

Let $G: X \rightarrow X$, then a point $x^{*} \in X$ such that $G\left(x^{*}\right)=x^{*}$ is a fixed point of $G$. The following theorem provides sufficient condition for the existence and uniqueness of a fixed point [13]. Moreover, if mapping that generates the iteration is a contraction, it ensures the stability of the iteration.

Theorem 1 (Banach's fixed point theorem). If $G: X \rightarrow X$ is a contraction mapping on a nonempty complete metric space $(X, d)$, then $G$ has only one fixed point $x^{*}$. Moreover, $x^{*}$ can be found as follows: start with an arbitrary $x_{0} \in X$ and define the sequence $x_{n+1}=G\left(x_{n}\right)$, then $\lim _{n \rightarrow \infty} x_{n}=x^{*}$.

Definition 2. Let $x^{*}$ be a fixed point of the iteration $x_{n+1}=G\left(x_{n}\right)$. $x^{*}$ is locally asymptotically stable if there exist a neighborhood $U$ of $x^{*}$, such that for each starting value $x_{0} \in U$ we get that

$$
\lim _{n \rightarrow \infty} x_{n}=x^{*}
$$


If this neighborhood $U$ is the entire domain of $G$, then $x^{*}$ is a globally asymptotically stable fixed point.

Corollary 1. If $G: X \rightarrow X$ is a contraction mapping on a nonempty complete metric space $(X, d)$, then its unique fixed point $x^{*}$ is globally asymptotically stable.

The following property of the sigmoid function will be applied: The derivative of the sigmoid function $f: \mathbb{R} \rightarrow \mathbb{R}, f(x)=1 /\left(1+e^{-\lambda x}\right),(\lambda>0)$ is bounded by $\lambda / 4$. Moreover, for every $x, y \in \mathbb{R}$ the following inequality holds

$$
|f(x)-f(y)| \leq \lambda / 4 \cdot|x-y| \text {. }
$$

Basic properties of the spectral radius of a matrix $M[6]$ :

- The spectral radius of matrix $M \in \mathbb{R}^{n \times n}$ is given by

$$
\rho(M)=\max \left\{\left|\lambda_{i}\right|: \lambda_{i} \text { eigenvalue of } M\right\}
$$

We should note that the spectral radius itself is not a norm.

- $\rho(M)=\inf \left\{\|M\|:\|*\|\right.$ is a matrix norm on $\left.\mathbb{R}^{n \times n}\right\}$

- Let matrix $M$ have spectral radius $\rho(M)$. If $\varepsilon>0$ is any positive number, then there exists a matrix norm $\|\cdot\|$, such that $\rho(M) \leq\|M\| \leq \rho(M)+\varepsilon$.

- If for matrices $M_{1}, M_{2}$ the entry-wise inequality $0 \leq M_{1} \leq M_{2}$ holds, then $\rho\left(M_{1}\right) \leq \rho\left(M_{2}\right)$.

\section{Input-Output Fuzzy Cognitive Maps}

In classical fuzzy cognitive map modelling, the concepts have their initial activation values and the final activation values are computed as the limit of the iteration (if the limit exists). In some cases, few features of the modelled system should not change during the simulation. From the FCM point of view, it means that values of some concepts should not change, but must remain the same for all steps of the iteration. This fact requires the re-thinking of the FCM-based modelling.

Based on the well-known discrete time linear time-invariant model:

$$
\begin{aligned}
& x(k+1)=A x(k)+B u(k) \\
& y(k+1)=C x(k)+D u(k)
\end{aligned}
$$

Groumpos, Anninou et al. introduced the following FCM model $[1,3]$ :

$$
\begin{aligned}
& x(k+1)=f\left(W_{A} x(k)+W_{B} u(k)\right) \\
& y(k+1)=f\left(W_{C} x(k)+W_{D} u(k)\right)
\end{aligned}
$$

where $u \in \mathbb{R}^{r}, x \in \mathbb{R}^{p}, y \in \mathbb{R}^{m}$. The matrices are extracted from the weight structure of the FCM: 
- $W_{A}$ describes the dynamics between the states $(x)$;

- $W_{B}$ describes the role of the inputs $(u)$;

- $W_{C}$ describes the role of $x$ in the output $(y)$;

- $W_{D}$ describes the contribution of $u$ to the output.

The following block scheme defines the weight matrix. The order of concepts: input, state, output (vertically and horizontally). Of course it is a bit redundant modell, since a state neuron can have input and state can be an output, too, but for the analogy with discrete linear systems we preserve these categories.

$$
W=\left[\begin{array}{c|c|c}
0 & 0 & 0 \\
\hline W_{B} & W_{A} & 0 \\
\hline W_{D} & W_{C} & 0
\end{array}\right]
$$

The activation vector $A \in \mathbb{R}^{r+p+m}$ then $A=[u, x, y]^{T}$. The updating process is determined by the mapping $A(k+1)=G(A(k))$, where $G: \mathbb{R}^{r+p+m} \rightarrow \mathbb{R}^{r+p+m}$ is defined elementwise:

- Input variables: if $1 \leq i \leq r$, then $A_{i}=u_{i}$ (constant input signal for every input channel);

- State variables: if $r+1 \leq i \leq r+p$, then

$$
A_{i}(k+1)=f\left(w_{A i} x(k)+w_{B i} u\right)=f\left(w_{i} A(k)\right)
$$

- Output variables: if $r+p+1 \leq i \leq r+p+n$, then

$$
A_{i}(k+1)=f\left(w_{C i} x(k)+w_{D i} u\right)=f\left(w_{i} A(k)\right)
$$

Consequently,

$$
\begin{aligned}
A(k+1) & =[\underbrace{A_{1}, \ldots, A_{r}}_{\text {input }}, \underbrace{A_{r+1}, \ldots, A_{r+p}}_{\text {state }}, \underbrace{A_{r+p+1}, \ldots, A_{r+p+m}}_{\text {output }}]^{T} \\
& =[\underbrace{A_{1}, \ldots, A_{r}}_{u}, \underbrace{f\left(w_{A i} x(k)+w_{B i} u\right)}_{r+1 \leq i \leq r+p}, \underbrace{f\left(w_{C i} x(k)+w_{D i} u\right)}_{r+p+1 \leq i \leq r+p+m}]^{T}
\end{aligned}
$$

\subsection{Convergence Condition for the Input-Output FCM}

In this subsection, a sufficient condition for the existence and uniqueness of fixed points of input-output FCMs will be stated. The fixed point is unique in the sense that for a given input, the FCM reaches the same fixed point (activation vector) regardless of the initial values of the other (state and output) concepts. 
The condition is based on the Jacobian matrix (matrix of the partial derivatives) of the mapping $G$ generating the iteration and the on the tight upper bound of the derivative of the sigmoid function.

The Jacobian of mapping $G$ is the matrix $J_{G}(i, j)=\frac{\partial G_{i}}{\partial A_{j}}$, namely

- for input variables $(u)$ :

$$
J_{G}(i, j)=\left\{\begin{array}{l}
1 \text { if } i=j \\
0 \text { otherwise }
\end{array}\right.
$$

- for state variables $(x)$ :

$$
J_{G}(i, j)=\left\{\begin{array}{l}
\lambda \cdot w_{B i j} f\left(w_{i} A\right)\left(1-f\left(w_{i} A\right)\right), \text { if }(i, j) \in(\text { state, } \text { input }) \\
\lambda \cdot w_{A i j} f\left(w_{i} A\right)\left(1-f\left(w_{i} A\right)\right), \text { if }(i, j) \in(\text { state, state })
\end{array}\right.
$$

- for output variables $(y)$ :

$$
J_{G}(i, j)=\left\{\begin{array}{l}
\lambda \cdot w_{D i j} f\left(w_{i} A\right)\left(1-f\left(w_{i} A\right)\right), \text { if }(i, j) \in \text { (output, input) } \\
\lambda \cdot w_{C i j} f\left(w_{i} A\right)\left(1-f\left(w_{i} A\right)\right), \text { if }(i, j) \in \text { (output, state) }
\end{array}\right.
$$

Since the input terms are constant values (do not change during the iteration), the iteration is convergent if and only if the dynamical terms generate a convergent sequence. The dynamics of this part is governed by the submatrix

$$
W^{\prime}=\left[\begin{array}{l|l}
W_{B} & W_{A} \\
\hline W_{D} & W_{C}
\end{array}\right]
$$

The Jacobian of $G$ belonging to this submatrix is

$$
J_{G}^{\prime}=\lambda \cdot \operatorname{diag}\left[f\left(w_{i} A\right)\left(1-f\left(w_{i} A\right)\right)\right] \cdot W^{\prime}
$$

Since for any $A$ and $w_{i}, f\left(w_{i} A\right)\left(1-f\left(w_{i} A\right)\right) \leq 1 / 4$, the spectral radius of the Jacobian at any point:

$$
\rho\left(J_{G}^{\prime}\right) \leq \rho\left(\frac{\lambda}{4} W^{\prime}\right)=\frac{\lambda}{4} \rho\left(W^{\prime}\right)
$$

If the spectral radius over the whole space is less than one, then the iteration converges to a unique fixed point. So we can conclude the following theorem:

Theorem 2. Consider the input-output FCM model described by Eq.9 with a constant input vector $u$. Let $W^{\prime}$ be the matrix constructed by matrices $W_{A}, W_{B}, W_{C}$ and $W_{D}$, according to Eq. 12. If

$$
\rho\left(W^{\prime}\right)<\frac{4}{\lambda}
$$

then the iteration converges to a unique fixed point, regardless of the initial activation values of the state and output concepts. 
The spectral radius of a matrix is less than any norm of this matrix, i.e. $\rho\left(W^{\prime}\right) \leq\left\|W^{\prime}\right\|$. It means that if we express a condition for convergence to a unique stable equlibrium point using any norm of $W^{\prime}$, then we get weaker theorem. Nevertheless, in some cases a weaker a condition gives more comprehensible explanation.

Remark 1. We have concluded that if $\rho\left(W^{\prime}\right)<\frac{4}{\lambda}$, then the FCM has exactly one fixed point, i.e. the limit of the iteration process is the same, regardless to the initial values of non-input variables. It also means that this fixed point is globally asymptotically stable.

\subsection{Further Convergence Conditions}

In this subsection, we prove other conditions for the convergence of input-output FCMs. Although these conditions are weaker, they might be useful, since they are directly based on the weight structure of the FCM. First, we recall some definitions about the structure of the network.

Definition 3. The weighted in-degree of concept $C_{j}$ equals the sum of the absolute values of the weights of in-coming edges:

$$
\operatorname{deg}_{j}^{i n}=\sum_{i=1}^{n}\left|w_{i j}\right|
$$

which is the sum of the absolute values of the entries of the jth column of $W$.

Definition 4. The weighted out-degree of concept $C_{i}$ equals the sum of the absolute values of the weights of out-going edges:

$$
\operatorname{deg}_{i}^{\text {out }}=\sum_{j=1}^{n}\left|w_{i j}\right|
$$

which is the sum of the absolute values of the entries of the ith row of $W$.

Although usually not considered graphically as a real edge, but self-feedback means self-loop in the graph. So if self-feedbacks are applied in the iteration, then the weights of the feedback are counted in the in-degree and the out-degree, too. It is the reason that we did not exclude $i=j$ from the summations above.

Definition 5. The connectivity of an FCM is the ratio of the number of connections between concepts to the maximum number of such possible connections.

In some sense, connectivity measures the 'density' of the network. If self-feedback is allowed, then the maximum number of connections is $n^{2}$, if not, then the maximum number of connections is $n(n-1)$.

Definition 6. The weighted connectivity of an FCM is the ratio of the sum of absolute values of weights of connections between concepts to the maximum number of such possible connections. 
If self-feedback is allowed, then the weighted connectivity is

$$
\operatorname{Con}_{w}=\frac{\sum_{i=1}^{n} \sum_{j=1}^{n}\left|w_{i j}\right|}{n^{2}}
$$

If self-feedback is not allowed, then the weighted connectivity is

$$
\operatorname{Con}_{w}=\frac{\sum_{i=1}^{n} \sum_{j=1}^{n}\left|w_{i j}\right|}{n(n-1)}
$$

Theorem 3. Let $\lambda$ be the parameter of the sigmoid threshold function applied for every concept. If the maximal in-degree of the FCM (including possible feedback) is less than $4 / \lambda$, then the FCM has one and only one fixed point.

Proof. Using the definition of in-degree:

$$
\max _{1 \leq j \leq n} \operatorname{deg}_{j}^{i n}=\max _{1 \leq j \leq n} \sum_{i=1}^{n}\left|w_{i j}\right|=\|W\|_{1}
$$

Since $\|W\|_{1} \geq \rho(W)$, if $\|W\|_{1}<4 / \lambda$, then $\rho(W)<4 / \lambda$, which ensures the convergence to a unique fixed point.

Theorem 4. Let $\lambda$ be the parameter of the sigmoid threshold function applied for every concept. If the maximal out-degree of the FCM (including possible feedback) is less than $4 / \lambda$, then the FCM has one and only one fixed point.

Proof. The proof goes similarly to the previous one, but instead of 1-norm we use the infinity norm.

$$
\max _{1 \leq i \leq n} d e g_{i}^{\text {out }}=\max _{1 \leq i \leq n} \sum_{j=1}^{n}\left|w_{i j}\right|=\|W\|_{\infty}
$$

As in the previous case, if $\|W\|_{\infty}<4 / \lambda$, then $\rho(W)<4 / \lambda$, which ensures the convergence to a unique fixed point.

Theorem 5. Let $\lambda$ be the parameter of the sigmoid threshold function applied for every concept. If the weighted connectivity $\left(C_{\text {Con }}\right)$ of the FCM small enough, namely

1. if self-feedback is allowed:

$$
\operatorname{Con}_{w}<\frac{4}{\lambda n^{2}},
$$

2. if self-feedback is not allowed:

$$
\operatorname{Con}_{w}<\frac{4}{\lambda n(n-1)},
$$

then the FCM has one and only one fixed point. 
Proof. Consider the following entry-wise matrix norm:

$$
\sum_{i=1}^{n} \sum_{j=1}^{n}\left|w_{i j}\right|
$$

(Unfortunately, the usual notation of this norm is $\|*\|_{1}$, which is confusing, since the 1-norm has the same notation.) We know that

$$
\rho(W) \leq \sum_{i=1}^{n} \sum_{j=1}^{n}\left|w_{i j}\right|
$$

So, if $\sum_{i=1}^{n} \sum_{j=1}^{n}\left|w_{i j}\right|<4 / \lambda$, then $\rho(W)<4 / \lambda$.

Consequently, if $\frac{\lambda}{4} \sum_{i=1}^{n} \sum_{j=1}^{n} w_{i j}<1$, then the mapping is a contraction. It means that the iteration converges to a unique fixed point, regardless to the initial value. Rearranging this inequality and division both sides by $n^{2}$ ( or $n(n-1)$ ) completes the proof.

The direct practical usability of this result is very limited, since it gives very weak condition. Nevertheless, it has an important mathematical statement: extremely weakly connected fuzzy cognitive maps always produce simple behaviour. Of course, the notion 'weakly' depends on $n$ and $\lambda$.

\subsection{Different Input - Different Output?}

Under certain circumstances, classical FCMs may converge to the same equilibrium state (fixed point) from completely different initial values. This property is advantageous in some applications, for example, it ensures the system's robustness against noise, while it is not useful for example in pattern recognition problems. In this subsection, we examine input-output FCMs from this point of view.

Let us assume that the inputs are $u_{1}$ and $u_{2}$, and the iteration converges to a fixed point in both cases. Let's denote these fixed points by $A_{1}^{*}$ and $A_{2}^{*}$, respectively. According to our assumption, both scenario lead to a steady state, i.e.:

$$
\begin{aligned}
& A_{1}^{*}=\left[u_{1}, x_{1}^{*}, y_{1}^{*}\right]^{T} \in \mathbb{R}^{r+p+m} \\
& A_{2}^{*}=\left[u_{2}, x_{2}^{*}, y_{2}^{*}\right]^{T} \in \mathbb{R}^{r+p+m}
\end{aligned}
$$

Consequently, the steady state equations hold for $\left[u_{1}, x_{1}^{*}, y_{1}^{*}\right]^{T}$ and $\left[u_{2}, x_{2}^{*}, y_{2}^{*}\right]^{T}$ :

$$
\begin{array}{ll}
x_{1}^{*}=f\left(W_{A} x_{1}^{*}+W_{B} u_{1}\right) & x_{2}^{*}=f\left(W_{A} x_{2}^{*}+W_{B} u_{2}\right) \\
y_{1}^{*}=f\left(W_{C} x_{1}^{*}+W_{D} u_{1}\right) & y_{2}^{*}=f\left(W_{C} x_{2}^{*}+W_{D} u_{2}\right)
\end{array}
$$


Let's assume, that $u_{1} \neq u_{2}$, but $x_{1}^{*}=x_{2}^{*}$. From the equations and from the monotonicity of $f$ we have

$$
W_{A} x_{1}^{*}+W_{B} u_{1}=W_{A} x_{2}^{*}+W_{B} u_{2}
$$

Rearranging the equation yields:

$$
W_{A}\left(x_{1}^{*}-x_{2}^{*}\right)=W_{B}\left(u_{2}-u_{1}\right)
$$

According to our assumption, the left hand side is zero:

$$
0=W_{B}\left(u_{2}-u_{1}\right)
$$

Since $u_{1} \neq u_{2}$, this equality holds if and only if $u_{2}-u_{1}$ lies in the null-space of $W_{B}$. If $W_{B}$ is of full rank, then $\operatorname{dim} \operatorname{Ker} W_{B}=0$, so every different input value generates different steady-state values $\left(\operatorname{Ker} W_{B}\right.$ denotes the null-space, a.k.a. kernel of $\left.W_{B}\right)$. When $\operatorname{dim} \operatorname{Ker} W_{B} \neq 0$, and $u_{1}-u_{2} \in \operatorname{Ker} W_{B}$, then $u_{1}$ and $u_{2}$ generate the same equilibrium state. Else, when $u_{1}-u_{2} \notin \operatorname{Ker} W_{B}$, they produce different $x_{1}^{*}$ and $x_{2}^{*}$. Similar arguments hold for $y_{1}^{*}$ and $y_{2}^{*} \cdot y_{1}^{*}=y_{2}^{*}$ implies that $u_{2}-u_{1}$ lies in the null-space of $W_{D}$, but there are infinite number of cases when $u_{1}-u_{2} \notin K e r W_{D}$, and in these cases $u_{1} \neq u_{2}$ yields $y_{1}^{*} \neq y_{2}^{*}$.

\section{Hybrid Fuzzy Cognitive Maps}

An other input-output model has been introduced by Napoles et al. [9] under the name hybrid FCM, with the following more general and highly flexible sigmoid threshold function defined for the $i$ th concept :

$$
f_{i}(x)=l_{i}+\frac{u_{i}-l_{i}}{1+e^{-\lambda_{i}\left(x-h_{i}\right)}}
$$

The topology of the proposed neural system is comprised of $r$ input neurons and $m$ output neurons, so there are no distinct inner state neurons. The weight matrix $W$ is composed of two submatrices $W_{I}$ and $W_{O}$. The first one contains the connections between the input concepts, while the second one contains the weights connecting the input neurons with the output ones. There are no connections from output neurons to input neurons.

Comparing this model to the previous one, we can observe that

- here is no difference between input and state concepts;

- inputs do not act directly on the output;

- the transfer functions $f_{i}$ are highly customized to each neuron, ensuring more flexibility in modelling.

$$
W=\left[\begin{array}{l|l}
W_{I} & 0 \\
\hline W_{O} & 0
\end{array}\right]
$$


The dynamics of the system is determined by the input part $W_{I}$ (but don't forget that in this model there is no difference between input and state concepts).

The general term of the Jacobian of the mapping that generates the updating process is the following:

$$
J_{G}(i, j)=\frac{\partial G_{i}}{\partial A_{j}}=w_{i j} \lambda_{i}\left(u_{i}-l_{i}\right) \frac{1}{1+e^{-\lambda_{i}\left(w_{i} A-h_{i}\right)}}\left(1-\frac{1}{1+e^{-\lambda_{i}\left(w_{i} A-h_{i}\right)}}\right)
$$

With the shorthand $g_{i}=\frac{1}{1+e^{-\lambda_{i}\left(w_{i} A-h_{i}\right)}}$, the Jacobian is

$$
J_{G}=\operatorname{diag}\left[\lambda_{i}\left(u_{i}-l_{i}\right)\right] \operatorname{diag}\left[g_{i}\left(1-g_{i}\right)\right] \cdot W
$$

Since $g_{i}\left(1-g_{i}\right) \leq 1 / 4$, the following inequality holds for the spectral radius of the Jacobian at any point:

$$
\rho\left(J_{G}\right) \leq \frac{1}{4} \rho\left(\operatorname{diag}\left[\lambda_{i}\left(u_{i}-l_{i}\right)\right] \cdot W\right)
$$

Moreover, because of the block structure of $W$, the spectral radius (largest absolute value of the eigenvalues) of $W$ equals the spectral radius of $W_{I}$ (it also proves that the dynamics of the system is determined by the input neurons and their weight structure). Consequently,

$$
\rho\left(J_{G}\right) \leq \frac{1}{4} \rho\left(\operatorname{diag}\left[\lambda_{i}\left(u_{i}-l_{i}\right)\right] \cdot W_{I}\right)
$$

Similarly to the previous section, we get the following theorem:

Theorem 6. Consider an FCM with weight structure described by Eq. 32 and transfer functions defined by Eq.31. If

$$
\frac{1}{4} \rho\left(\operatorname{diag}\left[\lambda_{i}\left(u_{i}-l_{i}\right)\right] \cdot W_{I}\right)<1
$$

then the FCM has exactly one fixed point. This fixed point is the limit of the iteration from any starting point.

Remark 2. In a special case, when $l_{i}=-u_{i}$ and $h_{i} \equiv 0$, the concept vector $A=[0, \ldots, 0]^{T}$ is always a fixed point, but not always a fixed point attractor. If the inequality in Theorem 6 holds, then this point is a globally asymptotically stable equilibrium point. On the other hand, when the inequality does not hold, the iteration may lead to this fixed point from certain starting point(s) (these are the elements of $\operatorname{Ker} W$ ), but this fixed point is not stable. This problem was discussed for the case of hyperbolic tangent threshold function in [5].

\section{Summary}

In this paper, the input-output fuzzy cognitive map model has been examined from the viewpoint of unique fixed points. Based on the spectral radius 
of the weight matrix and with various matrix norms, several convergence conditions have been proved. Although the conditions expressed by matrix norms are weaker, they are might more understandable for the users of FCMs.

Classical FCMs may produce the same output for totally different initial activation values. Although this property is useful in some models, since it means a kind of robustness concerning noise, there are many applications (for example pattern recognition or classification problems), where this is a disadvantageous feature. As we have seen, the input-output model does not have this drawback, it can produce different fixed points for different outputs. On the other hand, there are cases when different input values yield the same output values.

Finally, convergence condition for another type of input-output FCM was introduced, expressed by the spectral radius of the submatrix containing the weight structure between the input neurons.

Acknowledgment. The research presented in this paper was carried out as part of the EFOP-3.6.2-16-2017-00016 project in the framework of the New Széchenyi Plan. The completion of this project is funded by the European Union and co-financed by the European Social Fund.

This research was supported in part by National Research, Development and Innovation Office (NKFIH) K124055.

\section{References}

1. Anninou, A.P., Groumpos, P.P., Poulios, P., Gkliatis, I.: A new approach of dynamic fuzzy cognitive knowledge networks in modelling diagnosing process of meniscus injury. IFAC PapersOnLine 50(1), 5861-5866 (2017). https://doi.org/ 10.1016/j.ifacol.2017.08.1289

2. Boutalis, Y., Kottas, T.L., Christodoulou, M.: Adaptive estimation of fuzzy cognitive maps with proven stability and parameter convergence. IEEE Trans. Fuzzy Syst. 17(4), 874-889 (2009). https://doi.org/10.1109/TFUZZ.2009.2017519

3. Groumpos, P.P., Anninou, A.P.: A critical overview of modelling methods and decision support systems for complex dynamic systems. Ann. Fac. Eng. Hunedoara Int. J. Eng. 15(3), 17-26 (2017)

4. Harmati, I.Á., Hatwágner, M.F., Kóczy, L.T.: On the existence and uniqueness of fixed points of fuzzy cognitive maps. In: Medina, J., et al. (eds.) IPMU 2018. CCIS, vol. 853, pp. 490-500. Springer, Cham (2018). https://doi.org/10.1007/978-3-31991473-2_42

5. Harmati, I.Á., Kóczy, L.T.: Notes on the dynamics of hyperbolic tangent fuzzy cognitive maps. In: 2019 IEEE International Conference on Fuzzy Systems (FUZZIEEE), pp. 1-6. IEEE (2019). https://doi.org/10.1109/FUZZ-IEEE.2019.8858950

6. Horn, R.A., Johnson, C.R.: Matrix Analysis, 2nd edn. Cambridge University Press, New York (2013). https://doi.org/10.1017/9781139020411

7. Knight, C.J., Lloyd, D.J., Penn, A.S.: Linear and sigmoidal fuzzy cognitive maps: an analysis of fixed points. Appl. Soft Comput. 15, 193-202 (2014). https://doi. org/10.1016/j.asoc.2013.10.030

8. Lee, I.K., Kwon, S.H.: Design of sigmoid activation functions for fuzzy cognitive maps via Lyapunov stability analysis. IEICE Trans. Inf. Syst. 93(10), 2883-2886 (2010). https://doi.org/10.1587/transinf.E93.D.2883 
9. Nápoles, G., Jastrzebska, A., Mosquera, C., Vanhoof, K., Homenda, W.: Deterministic learning of hybrid fuzzy cognitive maps and network reduction approaches. Neural Netw. 124, 258-268 (2020). https://doi.org/10.1016/j.neunet.2020.01.019

10. Nápoles, G., Papageorgiou, E., Bello, R., Vanhoof, K.: On the convergence of sigmoid fuzzy cognitive maps. Inf. Sci. 349-350, 154-171 (2016). https://doi.org/10. 1016/j.ins.2016.02.040

11. Nápoles, G., Papageorgiou, E., Bello, R., Vanhoof, K.: Learning and convergence of fuzzy cognitive maps used in pattern recognition. Neural Process. Lett. 45(2), 431-444 (2017). https://doi.org/10.1007/s11063-016-9534-x

12. Papageorgiou, E.I., Salmeron, J.L.: Methods and algorithms for fuzzy cognitive map-based decision support. In: Papageorgiou, E.I. (ed.) Fuzzy Cognitive Maps for Applied Sciences and Engineering (2013). https://doi.org/10.1007/978-3-64239739-4_1

13. Rudin, W., et al.: Principles of Mathematical Analysis, vol. 3. McGraw-Hill, New York (1964)

14. Stylios, C.D., Groumpos, P.P.: Modeling complex systems using fuzzy cognitive maps. IEEE Trans. Syst. Man Cybern. Part A Syst. Hum. 34(1), 155-162 (2004). https://doi.org/10.1109/TSMCA.2003.818878

15. Tsadiras, A.K.: Comparing the inference capabilities of binary, trivalent and sigmoid fuzzy cognitive maps. Inf. Sci. 178(20), 3880-3894 (2008). https://doi.org/ 10.1016/j.ins.2008.05.015 